\title{
675.
}

\section{ON THE FLEFLECNODAL PLANES OF A SURFACE.}

[From the Quarterly Journal of Pure and Applied Mathematics, vol. xv. (1878), pp. $49-51$.

IF at a node (or double point) of a plane curve there is on one of the branches an inflexion, (that is, if the tangent has a 3-pointic intersection with the branch), the node is said to be a flecnode; and if there is on each of the branches an inflexion, then the node is said to be a fleflecnode. The tangent plane of a surface intersects the surface in a plane curve having at the point of contact a node; if this is a flecnode or a fleflecnode, the tangent plane is said to be a flecnodal or a fleflecnodal plane accordingly. For a quadric surface each tangent plane is fleflecnodal; this is obvious geometrically (since the section is a pair of lines), and it will presently appear that the analytical condition for such a plane is satisfied. In fact, if the origin be taken at a point of a surface, so that $z=0$ shall be the equation of the tangent plane, then in the neighbourhood of the point we have

$$
z=(x, y)^{2}+(x, y)^{3}+\& c .
$$

and the condition for a fleflecnodal plane is that the term $(x, y)^{2}$ shall be a factor of the succeeding term $(x, y)^{3}$. Now for a quadric surface the equation is

that is,

$$
z=\frac{1}{2}\left\{a x^{2}+2 h x y+b y^{2}+2(f y+g x) z+c z^{2}\right\}
$$

$$
z\left(1-f y-g x-\frac{1}{2} c z\right)=\frac{1}{2}\left(a x^{2}+2 h x y+b y^{2}\right),
$$

or developing as far as the third order in $(x, y)$, we have

$$
z=\frac{1}{2}\left(a x^{2}+2 h x y+b y^{2}\right)(1+f y+g x)
$$

so that the condition in question is satisfied. 
In what follows, I take for greater simplicity $h=0$, (viz. $x=0, y=0$ are here the tangents to the two curves of curvature at the point in question), and to avoid fractions write $2 f, 2 g$ in place of $f, g$ respectively; the developed equation of the quadric surface is thus

$$
z=\frac{1}{2}\left(a x^{2}+b y^{2}\right)+\left(a x^{2}+b y^{2}\right)(g x+f y) .
$$

I consider the parallel surface, obtained by measuring off on the normal a constant length $k$. If, as usual, $p, q$ denote $\frac{d z}{d x}$ and $\frac{d z}{d y}$ respectively, then, in general, $(X, Y, Z)$ being the coordinates of the point on the parallel surface,

But in the present case

$$
\begin{aligned}
& Z=z+\frac{k}{\sqrt{\left(1+p^{2}+q^{2}\right)}} \\
& X=x-\frac{k p}{\sqrt{\left(1+p^{2}+q^{2}\right)}} \\
& Y=y-\frac{k q}{\sqrt{\left(1+p^{2}+q^{2}\right)}}
\end{aligned}
$$

whence

$$
\begin{aligned}
& p=a x+3 a g x^{2}+2 a f x y+b g y^{2}, \\
& q=b y+a f x^{2}+2 b g x y+3 b f y^{2}
\end{aligned}
$$

$$
\begin{aligned}
& X=x-k\left(a x+3 a g x^{2}+2 a f x y+b g y^{2}\right), \\
& Y=y-k\left(b y+a f x^{2}+2 b g x y+3 b f y^{2}\right)
\end{aligned}
$$

or, putting for convenience,

$$
X=(1-k a) \xi, \quad Y=(1-k b) \eta,
$$

then, for a first approximation $x=\xi, y=\eta$; whence, writing

we find

$$
\begin{aligned}
& P=3 a g \xi^{2}+2 a f \xi \eta+b g \eta^{2} \\
& Q=a f \xi^{2}+2 b g \xi \eta+3 b f \eta^{2},
\end{aligned}
$$

and thence

$$
x=\xi+\frac{k P}{1-k a}, \quad y=\eta+\frac{k Q}{1-k b},
$$

Hence

$$
\begin{aligned}
p=a\left(\xi+\frac{k}{1+k a} P\right)+P & =a \xi+\frac{P}{1-k a}, \\
q & =b \eta+\frac{Q}{1-k b} .
\end{aligned}
$$

$$
\begin{aligned}
Z=\frac{1}{2}\left(a \xi^{2}+b \eta^{2}\right)+\frac{k a}{1-k a} \xi P & +\frac{k b}{1-k b} \eta Q+\left(a \xi^{2}+b \eta^{2}\right)(g \xi+f \eta) \\
& +k\left\{1-\frac{1}{2}\left(a^{2} \xi^{2}+b^{2} \eta^{2}\right)-\frac{a \xi P}{1-k a}-\frac{b \eta Q}{1-k b}\right\}
\end{aligned}
$$

or, finally,

$$
Z-k=\frac{1}{2}\left\{a(1-k a) \xi^{2}+b(1-k b) \eta^{2}\right\}+\left(a \xi^{2}+b \eta^{2}\right)(g \xi+f \eta),
$$


where, changing the origin to the point $x=0, y=0, z=k$ on the parallel surface, the coordinates of the consecutive point are $Z-k, X,=(1-k a) \xi$, and $Y,=(1-k b) \eta$.

We cannot, by any determination of the value of $k$, make the plane $\boldsymbol{Z}-k=0$ a fleflecnodal plane of the parallel surface; but if

then

$$
k=\frac{a f^{2}+b g^{2}}{a^{2} f^{2}+b^{2} g^{2}},
$$

$$
1-k a=\frac{b g^{2}(b-a)}{a^{2} f^{2}+b^{2} g^{2}}, \quad 1-k b=\frac{a f^{2}(a-b)}{a^{2} f^{2}+b^{2} g^{2}},
$$

and the equation becomes

$$
Z-k=\frac{1}{2} \frac{a b(b-a)}{a^{2} f^{2}+b^{2} g^{2}}\left(g^{2} \xi^{2}-f^{2} \eta^{2}\right)+\left(a \xi^{2}+b \eta^{2}\right)(g \xi+f \eta) ;
$$

viz. the term of the second has here a factor $g \xi+f \eta$ which divides the term of the third order, and the plane $Z-k=0$ is a flecnodal plane of the parallel surface. 